\title{
Bulk Viscous Anisotropic Cosmological Models with Generalized Chaplygin Gas with Time Varying Gravitational and Cosmological Constants
}

\author{
Shubha Kotambkar ${ }^{1}$, Gyan Prakash Singh², Rupali Kelkar ${ }^{3}$ \\ ${ }^{1}$ Department of Applied Mathematics, Laxminarayan Institute of Technology, Rashtrasant Tukadoji Maharaj \\ Nagpur University, Nagpur, India \\ ${ }^{2}$ Department of Mathematics, Visvesvaraya National Institute of Technology, Nagpur, India \\ ${ }^{3}$ Department of Applied Mathematics, S. B. Jain Institute of Technology, Management and Research, Nagpur, India \\ Email: shubha.kotambkar@rediffmail.com, gpsingh@mth.vnit.ac.in, rupali.kelkar@yahoo.com
}

Received 28 April 2015; accepted 6 June 2015; published 9 June 2015

Copyright (C) 2015 by authors and Scientific Research Publishing Inc.

This work is licensed under the Creative Commons Attribution International License (CC BY).

http://creativecommons.org/licenses/by/4.0/

\section{(c) (i) Open Access}

\section{Abstract}

This paper is devoted to studying the generalized Chaplygin gas models in Bianchi type III spacetime geometry with time varying bulk viscosity, cosmological and gravitational constants. We are considering the condition on metric potential $\frac{\dot{R}_{1}}{R_{1}}=\frac{\dot{R}_{2}}{R_{2}}=\frac{m_{1}}{t^{n}}, \frac{\dot{R}_{3}}{R_{3}}=\frac{m_{2}}{t^{n}}$. Also to obtain deterministic models we have considered physically reasonable relations like $P=p+\Pi, \eta=\eta_{0} \rho^{r}$ and the equation of state for generalized Chaplygin gas given by $p=\frac{-B}{\rho^{\alpha}}$. A new set of exact solutions of Einstein's field equations has been obtained in Eckart theory, truncated theory and full causal theory. Physical behaviour of the models has been discussed.

\section{Keywords}

Bianchi Type III, Bulk Viscosity, Cosmological Constant, Gravitational Constant, Generalized Chaplygin Gas

\section{Introduction}

The motivation behind the stimulated interest in anisotropic cosmological models is experimental study of iso-

How to cite this paper: Kotambkar, S., Singh, G.P. and Kelkar, R. (2015) Bulk Viscous Anisotropic Cosmological Models with Generalized Chaplygin Gas with Time Varying Gravitational and Cosmological Constants. Natural Science, 7, $312-323$. 
tropy of the cosmic microwave background radiation and speculation about the amount of the helium formed at the early stages of the evolution of the universe. The existence of anisotropic stage of the universe is supported by experimental data and numbers of scientific arguments in the literature which is supposed to be phased out during evolution. The present day universe is isotropic and homogeneous. In understanding the behavior of universe at early stages, anisotropic cosmological models have played a significant role. Singh and Singh [1] [2] have obtained cosmological models for Bianchi type $\mathrm{V}, \mathrm{VI}_{0}$, III and Kantowski-Sachs space-times within framework of Lyra geometry. Bianchi type III cosmological model in $f(R, T)$ theory of gravity has been discussed by Reddy et al. [3]. Pradhan et al. [4] discussed anisotropic Bianchi type III string cosmological models in normal gauge for Lyra's manifold with electromagnetic field. Singh et al. [5] have investigated Bianchi type III cosmological models in Lyra's geometry in the presence of massive scalar field.

The astronomical observations of type Ia supernovae [6]-[10], galaxy red shift surveys [11], cosmic background radiation data [12] [13] and large scale structure [14] convincingly suggest that present universe is undergoing the accelerated phase of expansion. To understand this accelerated behavior of universe, cosmological constant played a significant role. A large cosmological constant at early epoch is the basis of the inflationary model and the much smaller cosmological constant at a much later epoch is suggested by current observations. In an attempt to solve the discrepancy between the cosmological constant inferred from observations and the vacuum energy density resulting from quantum field theories, many researchers have proposed cosmological models with time varying $\Lambda$. Sahni and Starobinski [15] have presented detailed discussion on current observational situation focusing on cosmological tests on $\Lambda$.

The idea of variability of $G$ originated with the work of Dirac [16], who for the first time drew the attention of the scientific community to the time varying $G$ in context of cosmological model. The theory of an expanding universe supports the idea of time-dependent gravitational constant. Time varying $G$ has many interesting consequences in astrophysics. It is shown that $G$ varying cosmology is consistent with whatsoever cosmological observations available at present [17]. Variability of $G$ is also supported by observational results coming up from Lunar Laser Ranging [18]. Anisotropic cosmological models with bulk viscosity, variable $G$ and $\Lambda$ have been investigated by Chakraborty and Roy [19]. Singh and Beesham [20] have discussed anisotropic Bianchi type V perfect fluid space-time with variable $G$ and $\Lambda$. Singh [21] has focused on Robertson-Walker model with variable cosmological term and gravitational constant in cosmological relativity theory. Khurshudyan et al. [22] have studied observational constraints on models of the universe with time-variable gravitational and cosmological constants along modified gravity theory.

In the literature it has been discussed that during the early stages of evolution of the universe, bulk viscosity could arise in many circumstances and could lead to an effective mechanism of galaxy formation [23]. To consider more realistic models one must take into account the viscosity mechanism, which has already attracted the attention of many researchers. Bulk viscosity leading to an accelerated phase of the universe today has been studied by Fabris et al. [24]. Singh et al. [25] have presented a number of classes of solutions of Einstein's field equations with variable $G$ and $\Lambda$ and bulk viscosity coefficient in the frame work of non-causal theory. Singh and Chaubey [26], and Singh and Baghel [27] [28] have discussed some Bianchi type models with bulk viscosity. Recently Kotambkar et al. [29] have investigated anisotropic cosmological models with quintessence considering the effect of bulk viscosity.

It has been observed that the universe has entered an acceleration phase and some exotic dark energy must presently dominate [30] [31]. This hypothetical form of energy that permeates all of space tends to increase the rate of expansion of the universe. Hence in order to explain recent cosmic observations, dark energy is considered as prime candidate. Chaplygin gas may be useful for describing dark energy because of its negative pressure. Chaplygin gas (CG) is referred as exotic fluid, as it has positive energy density but negative pressure. Due to effectiveness of CG in explaining the evolution of the universe, several generalizations of Chaplygin gas have been proposed in the literature [32]-[34]. The form of equation of state (EOS) of matter is generalized by adding an arbitrary constant with an exponent over the mass density, referred as generalized Chaplygin gas (GCG) [35] [36]. The form of EOS is modified by adding an ordinary matter field, matching the recent observational fallouts GCG referred as modified Chaplygin gas (MCG) [37]. Alcaniz et al. [38] have investigated cosmological models with high red shift objects and the generalized Chaplygin gas. Paul et al. [39] have studied observational constraints on modified Chaplygin gas. 


\section{Field Equation}

We consider the Bianchi type III metric in the form

$$
\mathrm{d} s^{2}=-\mathrm{d} t^{2}+R_{1}^{2} \mathrm{~d} x^{2}+R_{2}^{2} \mathrm{e}^{2 x} \mathrm{~d} y^{2}+R_{3}^{2} \mathrm{~d} z^{2} .
$$

$R_{1}, R_{2}, R_{3}$ are function of $\mathrm{t}$ alone.

For perfect fluid distribution Einstein's field equations with gravitation and cosmological constant may be written as

$$
R_{i j}-\frac{1}{2} R g_{i j}=-8 \pi G T_{i j}+\Lambda g_{i j} .
$$

where $G$ is gravitational constant, $\Lambda$ is cosmological constant, which are time dependent.

The energy momentum tensor $T_{i j}$ for viscous fluid distribution is given by

$$
T_{i j}=(\rho+P) u_{i} u_{j}+P g_{i j},
$$

where

$$
P=p+\Pi \text {. }
$$

where $p$ is equilibrium pressure, $\Pi$ is bulk viscous stress together with $u_{i} u^{j}=1$.

Einstein's filed Equation (2) for the metric (1) leads to

$$
\begin{gathered}
\frac{\ddot{R}_{2}}{R_{2}}+\frac{\ddot{R}_{3}}{R_{3}}+\frac{\dot{R}_{2} \dot{R}_{3}}{R_{2} R_{3}}=-8 \pi G(p+\Pi)+\Lambda, \\
\frac{\ddot{R}_{1}}{R_{1}}+\frac{\ddot{R}_{3}}{R_{3}}+\frac{\dot{R}_{1} \dot{R}_{3}}{R_{1} R_{3}}=-8 \pi G(p+\Pi)+\Lambda, \\
\frac{\ddot{R}_{1}}{R_{1}}+\frac{\ddot{R}_{2}}{R_{2}}+\frac{\dot{R}_{1} \dot{R}_{2}}{R_{1} R_{2}}=-8 \pi G(p+\Pi)+\Lambda, \\
\frac{\dot{R}_{1} \dot{R}_{2}}{R_{1} R_{2}}+\frac{\dot{R}_{2} \dot{R}_{3}}{R_{2} R_{3}}+\frac{\dot{R}_{1} \dot{R}_{3}}{R_{1} R_{3}}-\frac{1}{R_{1}^{2}}=8 \pi G \rho+\Lambda, \\
\frac{\dot{R}_{1}}{R_{1}}-\frac{\dot{R}_{2}}{R_{2}}=0 .
\end{gathered}
$$

where the over head dot denote differentiation with respect to time $t$. An additional equation for the time changes of $G$ and $\Lambda$ is obtained by divergence of the Einstein tensor, i.e. $\left(R_{i j}-\frac{1}{2} R g_{i j}\right)_{; j}=0$ which leads to $\left(8 \pi G T_{i j}-\Lambda g_{i j}\right)_{; j}=0$, yielding

$$
8 \pi \dot{G} \rho+\dot{\Lambda}+8 \pi G\left[\dot{\rho}+(\rho+p+\Pi)\left(\frac{\dot{R}_{1}}{R_{1}}+\frac{\dot{R}_{2}}{R_{2}}+\frac{\dot{R}_{3}}{R_{3}}\right)\right]=0 .
$$

Equation (10) splits into two equations as

$$
\begin{gathered}
\dot{\rho}+(\rho+p)\left(\frac{\dot{R}_{1}}{R_{1}}+\frac{\dot{R}_{2}}{R_{2}}+\frac{\dot{R}_{3}}{R_{3}}\right)=0, \\
\dot{\Lambda}+8 \pi \dot{G} \rho=-8 \pi G \Pi\left(\frac{\dot{R}_{1}}{R_{1}}+\frac{\dot{R}_{2}}{R_{2}}+\frac{\dot{R}_{3}}{R_{3}}\right) .
\end{gathered}
$$

For the full causal non-equilibrium thermodynamics the causal evolution equation for bulk viscosity is given by $[40]$ 


$$
\Pi+\tau \dot{\Pi}=-\eta\left(\frac{\dot{R}_{1}}{R_{1}}+\frac{\dot{R}_{2}}{R_{2}}+\frac{\dot{R}_{3}}{R_{3}}\right)-\frac{\varepsilon \tau \Pi}{2}\left(\frac{\dot{R}_{1}}{R_{1}}+\frac{\dot{R}_{2}}{R_{2}}+\frac{\dot{R}_{3}}{R_{3}}+\frac{\dot{\tau}}{\tau}-\frac{\dot{\eta}}{\eta}-\frac{\dot{T}}{T}\right) .
$$

$T \geq 0$ absolute temperature, $\eta$ is bulk viscosity coefficient which cannot become negative, $\tau$ denote the relaxation time for transient bulk viscous effects. Causality requires $\tau>0$. When $\varepsilon=0$, Equation (13) reduces to evolution equation for truncated theory. For $\varepsilon=1$ Equation (13) reduces to evolution equation for full causal theory and for $\tau=0$ Equation (13) reduces to evolution equation for non-causal theory (Eckart's theory).

\section{Cosmological Solutions}

Since there are five basic Equations (5)-(9) and eight unknowns viz. $R_{1}, R_{2}, R_{3}, p, \rho, G, \Lambda$ and $\Pi$ therefore three more physically plausible relations among these variables will be considered for solving the set of equations.

\section{Case I: Non-Causal Cosmological Solution}

For non causal solution $\tau=0$, therefore the evolution Equation (13) takes the form of

$$
\Pi=-\eta\left(\frac{\dot{R}_{1}}{R_{1}}+\frac{\dot{R}_{2}}{R_{2}}+\frac{\dot{R}_{3}}{R_{3}}\right)=-3 \eta H
$$

To find the complete solution of the system of equations, following relations are taken into consideration.

The power law relation for bulk viscosity is taken as

$$
\eta=\eta_{0} \rho^{r},
$$

$\eta_{0}>0$ and $r$ is constant.

The equation of state is

$$
p=\frac{-B}{\rho^{\alpha}}, \quad 0<\alpha \leq 1, B>0
$$

We assume the solution of the system in the form

$$
\frac{\dot{R}_{1}}{R_{1}}=\frac{\dot{R}_{2}}{R_{2}}=\frac{m_{1}}{t^{n}}, \quad \frac{\dot{R}_{3}}{R_{3}}=\frac{m_{2}}{t^{n}} .
$$

where $n$ is constant. On integrating Equation (17), we get

$$
R_{1}=R_{2}=a \exp \left\{\frac{m_{1} t^{1-n}}{1-n}\right\} \text { and } R_{3}=b \exp \left\{\frac{m_{2} t^{1-n}}{1-n}\right\}
$$

where $a$ and $b$ are constants of integration.

Using Equations (16) and (17) in (11), we obtain

$$
\dot{\rho}+\rho \frac{\left(2 m_{1}+m_{2}\right)}{t^{n}}=\frac{B\left(2 m_{1}+m_{2}\right)}{t^{n} \rho^{\alpha}},
$$

which on solving yields

$$
\rho=\left[B+C \exp \left\{\frac{-\left(2 m_{1}+m_{2}\right)(\alpha+1)}{1-n} t^{1-n}\right\}\right]^{\frac{1}{\alpha+1}},
$$

where $C$ is constant of integration.

From Equation (20) and Figure 1 one can see that energy density is decreasing with evolution of the universe which is in fair agreement with observations.

On differentiating Equation (20), one can get

$$
\dot{\rho}=\frac{-C\left(2 m_{1}+m_{2}\right)}{t^{n}} \exp \left\{\frac{-\left(2 m_{1}+m_{2}\right)(\alpha+1)}{1-n} t^{1-n}\right\}\left[B+C \exp \left\{\frac{-\left(2 m_{1}+m_{2}\right)(\alpha+1)}{1-n} t^{1-n}\right\}\right]^{\frac{-\alpha}{\alpha+1}}
$$




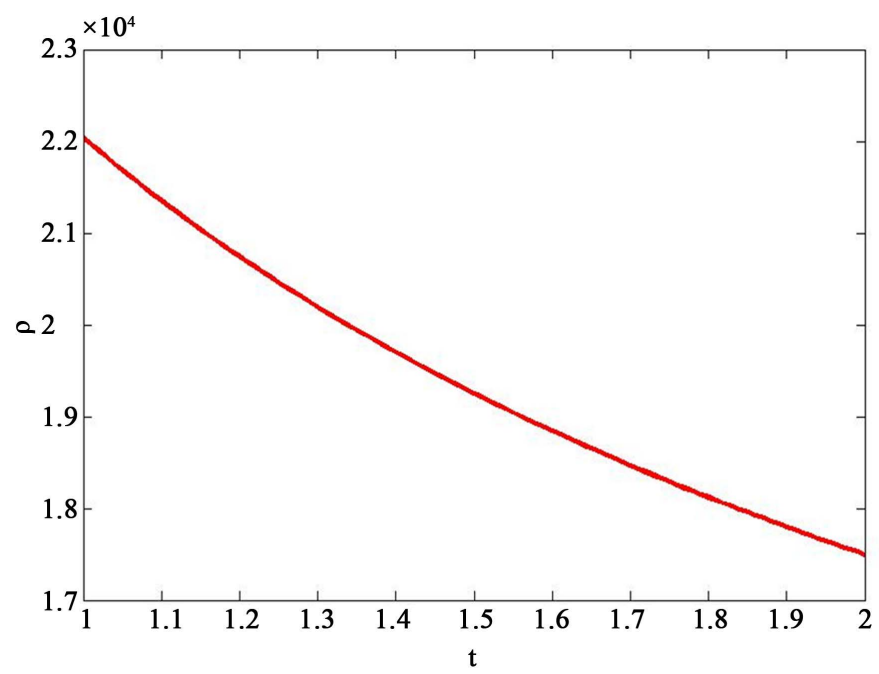

Figure 1. Shows variation of energy density $\rho$ with respect to cosmic time $t$. Here we consider $B=1, C=1, n=1.5, m_{1}=2, m_{2}=1$ and $\alpha=0.5$.

Now with the help of Equations (17) and (18), Equation (8) becomes

$$
\frac{m_{1}^{2}}{t^{2 n}}+\frac{m_{1} m_{2}}{t^{2 n}}+\frac{m_{1} m_{2}}{t^{2 n}}-\frac{1}{a^{2} \exp \left(\frac{-2 m_{1}}{1-n} t^{1-n}\right)}=8 \pi G \rho+\Lambda,
$$

which on differentiation leads to

$$
\frac{-2 n m_{1}\left(m_{1}+2 m_{2}\right)}{t^{2 n+1}}+\frac{2 m_{1}}{a^{2} t^{n}} \exp \left(\frac{-2 m_{1}}{1-n} t^{1-n}\right)=8 \pi \dot{G} \rho+8 \pi G \dot{\rho}+\dot{\Lambda} .
$$

Substituting Equations (12), (14) and (17) into Equation (23), we have

$$
\frac{-2 n m_{1}\left(m_{1}+2 m_{2}\right)}{t^{2 n+1}}+\frac{2 m_{1}}{a^{2} t^{n}} \exp \left(\frac{-2 m_{1}}{1-n} t^{1-n}\right)=8 \pi G \dot{\rho}+8 \pi G \eta\left(\frac{2 m_{1}+m_{2}}{t^{n}}\right)^{2} .
$$

By use of Equations (15) and (21), Equation (24) yields

$$
G=\frac{\left[C_{1} t^{-(2 n+1)}+2 m_{1} a^{-2} t^{-n} \exp \left(D_{1} t^{1-n}\right)\right]}{8 \pi\left[C_{2} t^{-n} \exp \left(D_{1} t^{1-n}\right)\left(B+C \exp \left(D t^{1-n}\right)\right)^{\frac{-\alpha}{\alpha+1}}+\left(2 m_{1}+m_{2}\right)^{2} t^{-2 n} \eta_{0}\left(B+C \exp \left(D t^{1-n}\right)\right)^{\frac{r}{\alpha+1}}\right]},
$$

where $D=\frac{-\left(2 m_{1}+m_{2}\right)(\alpha+1)}{1-n}, C_{1}=-2 n m_{1}\left(m_{1}+2 m_{2}\right), C_{2}=-C\left(2 m_{1}+m_{2}\right), D_{1}=\frac{-2 m_{1}}{1-n}$.

From Equation (25) and Figure 2 one can see that gravitational constant is increasing with evolution of the universe which goes with observations.

Using Equations (20) and (25), Equation (22) gives

$$
\begin{aligned}
\Lambda & =m_{1}\left(m_{1}+2 m_{2}\right) t^{-2 n}-a^{-2} \exp \left(D_{1} t^{1-n}\right) \\
& -\frac{\left[C_{1} t^{-(2 n+1)}+2 m_{1} a^{-2} t^{-n} \exp \left(D_{1} t^{1-n}\right)\right]}{\left[C_{2} t^{-n} \exp \left(D_{1} t^{1-n}\right)\left(B+C \exp \left(D t^{1-n}\right)\right)^{-1}+\left(2 m_{1}+m_{2}\right)^{2} t^{-2 n} \eta_{0}\left(B+C \exp \left(D t^{1-n}\right)\right)^{\frac{r-1}{\alpha+1}}\right]}
\end{aligned}
$$

From Equation (26) and Figure 3 one can see that cosmological constant is decreasing with evolution of the universe which is in fair agreement with observations. 


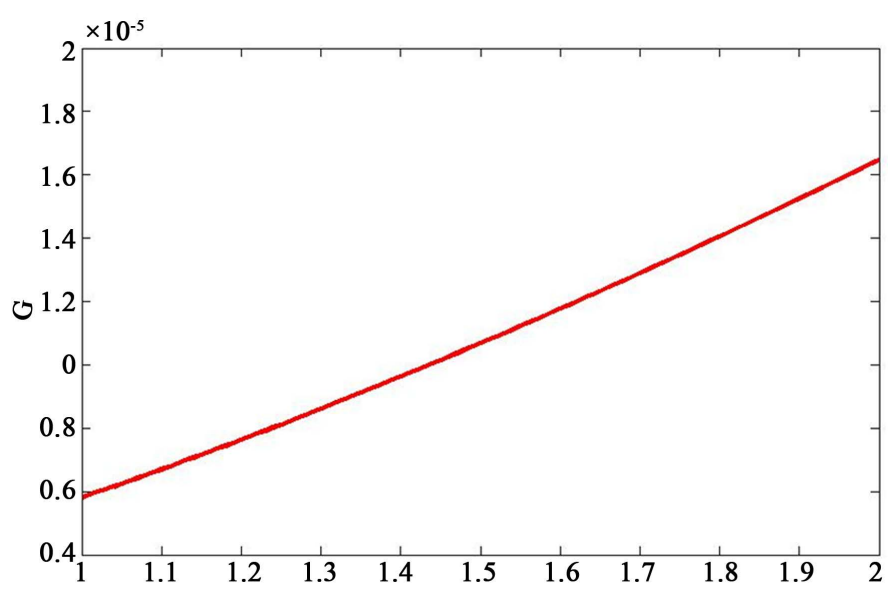

Figure 2. Shows variation of gravitational constant with respect to cosmic time $t$. Here we consider $B=1, C=1, n=1.5, m_{1}=2, m_{2}=1, \quad \alpha=0.5, r=1.5, a=1$ and $\eta_{0}=1$.

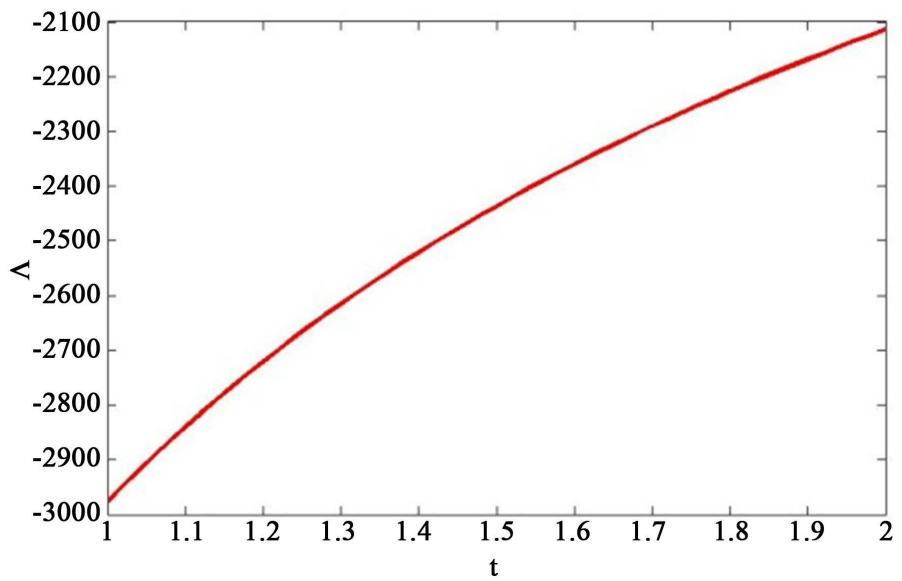

Figure 3. Shows variation of cosmological constant with respect to cosmic time $t$. Here we consider $B=1, C=1, n=1.5, m_{1}=2, m_{2}=1, \quad \alpha=0.5, r=1.5, a=1$ and $\eta_{0}=1$.

Now from Equations (15) and (20), we have

$$
\eta=\eta_{0}\left[B+C \exp \left(D t^{1-n}\right)\right]^{\frac{r}{\alpha+1}}
$$

From Equation (27) and Figure 4 one can see that bulk viscosity coefficient is decreasing with evolution of the universe which is in fair agreement with observations.

From Equations (14) and (17), the expression for bulk viscous stress is given by

$$
\Pi=\frac{-3 \eta_{0}\left(2 m_{1}+m_{2}\right)}{t^{n}}\left[B+C \exp \left(D t^{1-n}\right)\right]^{\frac{r}{\alpha+1}}
$$

Thus the metric (1) reduces to the form

$$
\mathrm{d} s^{2}=-\mathrm{d} t^{2}+a^{2} \exp \left\{\frac{-2 m_{1} t^{1-n}}{1-n}\right\}\left(\mathrm{d} x^{2}+\mathrm{e}^{2 x} \mathrm{~d} y^{2}\right)+b^{2} \exp \left\{\frac{2 m_{2} t^{1-n}}{1-n}\right\} \mathrm{d} z^{2}
$$

The shear scalar [41] may be defined as

$$
\sigma^{2}=\frac{1}{12}\left[\left(\frac{\dot{g}_{11}}{g_{11}}-\frac{\dot{g}_{22}}{g_{22}}\right)^{2}+\left(\frac{\dot{g}_{22}}{g_{22}}-\frac{\dot{g}_{33}}{g_{33}}\right)^{2}+\left(\frac{\dot{g}_{33}}{g_{33}}-\frac{\dot{g}_{11}}{g_{11}}\right)^{2}\right]
$$




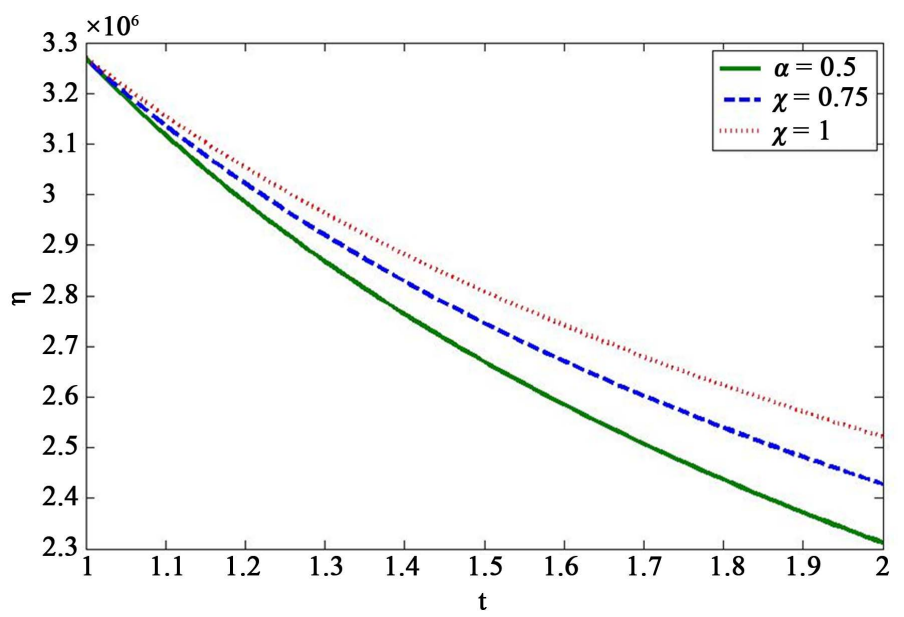

Figure 4. Shows variation of bulk viscosity coefficient with respect to cosmic time $t$. Here we consider $B=1, C=1, n=1.5, m_{1}=2, m_{2}=1, \alpha=0.5,0.75,1, r=1.5$.

For this model the Shear scalar is

$$
\sigma^{2}=\frac{2\left(m_{1}-m_{2}\right)^{2}}{3 t^{2 n}}
$$

From Equation (31) it is clear that as $t \rightarrow \infty$, shear dies out.

The expansion scalar is defined by

$$
\Theta=3 H, \quad H=\frac{\dot{R}}{R} .
$$

For this model expansion scalar is given by

$$
\Theta=\frac{2 m_{1}+m_{2}}{t^{n}}
$$

The deceleration parameter is related to the expansion scalar as

$$
q=-\frac{3 \dot{\Theta}+\Theta^{2}}{\Theta^{2}},
$$

For this model

$$
q=\frac{3 n}{2 m_{1}+m_{2}} t^{n-1}-1
$$

Foe accelerating expansion of the universe the deceleration parameter $q<0$ for $t<\left(\frac{2 m_{1}+m_{2}}{3 n}\right)^{\frac{1}{n-1}}$.

$$
\begin{gathered}
\text { Relative anisotropy }=\frac{\sigma^{2}}{\rho}=\frac{2\left(m_{1}-m_{2}\right)^{2}}{3 t^{2 n}}\left[B+C \exp \left\{\frac{-\left(2 m_{1}+m_{2}\right)(\alpha+1)}{1-n} t^{1-n}\right\}\right]^{\frac{-1}{\alpha+1}} \\
\frac{\sigma}{\Theta}=\frac{\sqrt{2}\left(m_{1}-m_{2}\right)}{\sqrt{3}\left(2 m_{1}+m_{2}\right)}=\text { constant }
\end{gathered}
$$

\section{Case II: Causal Cosmological Solution}

In addition to physically plausible relations (16), (17), in this case we assume

$$
\Lambda=\beta H^{2} .
$$


where $H$ is Hubble parameter, given by

$$
H=\frac{\dot{R}}{R} \text { and } R=\left(R_{1} R_{2} R_{3}\right)^{1 / 3} .
$$

From Equations (17) and (39), the Hubble parameter is given by

$$
H=\frac{2 m_{1}+m_{2}}{3 t^{n}}
$$

Using Equations (17)-(18), (38) and (40) in Equation (8), we get

$$
8 \pi G \rho=\frac{M}{t^{2 n}}-\frac{1}{a^{2}} \exp \left\{\frac{-2 m_{1} t^{1-n}}{1-n}\right\},
$$

where $M=m_{1}^{2}+2 m_{1} m_{2}-\frac{\beta}{9}\left(2 m_{1}+m_{2}\right)^{2}$.

From Equations (20) and (41),

$$
G=\frac{1}{8 \pi}\left[B+C \exp \left(D t^{1-n}\right)\right]^{\frac{-1}{\alpha+1}}\left[\frac{M}{t^{2 n}}-\frac{1}{a^{2}} \exp \left(D_{1} t^{1-n}\right)\right]
$$

From Equation (42) and Figure 5 one can see that gravitational constant is increasing with evolution of the universe which supports observations.

Substitute the values from Equations (17), (20), (38) and (42) in Equation (5), we get

$$
\Pi=\left[\frac{M_{1}}{t^{2 n}}-\frac{\left(m_{1}+m_{2}\right) n}{t^{n+1}}\right] \frac{-1}{8 \pi G}+\frac{B}{\rho^{\alpha}},
$$

where $M_{1}=m_{1}^{2}+m_{2}^{2}+m_{1} m_{2}-\frac{\beta}{9}\left(2 m_{1}+m_{2}\right)^{2}$.

By use of Equation (20), Equation (43) gives

$$
\begin{gathered}
\Pi=\frac{\left[C_{3} t^{n-1}-M_{1}\right]\left[B+C \exp \left(D t^{1-n}\right)\right]^{\frac{1}{\alpha+1}}}{[M-U(t)]}+\frac{B}{\left[B+C \exp \left(D t^{1-n}\right)\right]^{\frac{\alpha}{\alpha+1}}} \\
C_{3}=\left(m_{1}+m_{2}\right) n, U(t)=\frac{1}{a^{2}} t^{2 n} \exp \left\{D_{1} t^{1-n}\right\}
\end{gathered}
$$

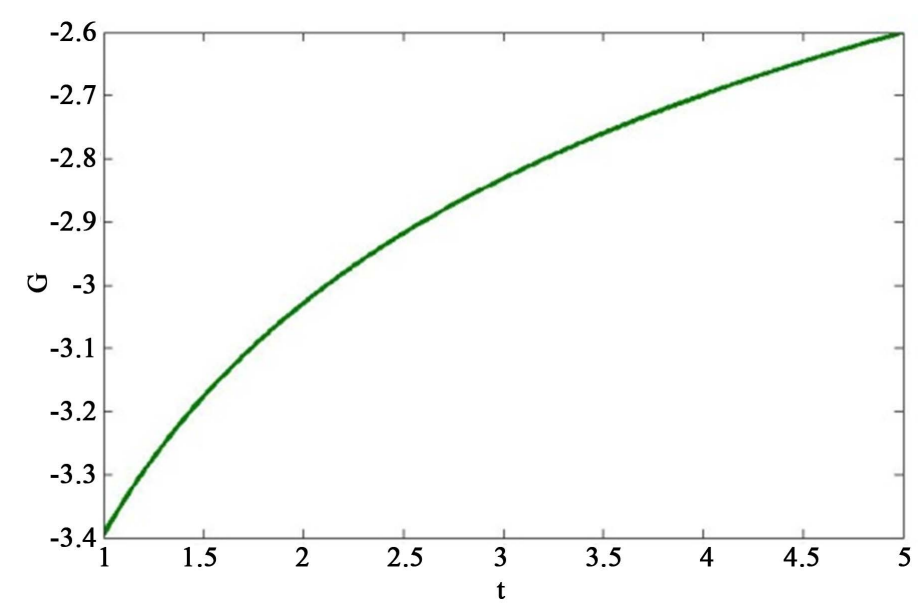

Figure 5. Shows variation of gravitational constant with respect to cosmic time $t$. Here we consider $B=1, C=1, n=1.5, m_{1}=2, m_{2}=1, \quad \alpha=0.5, r=1.5, a=1$ and $\eta_{0}=1$. 


\section{(i) Evaluation of Bulk viscosity in Truncated Causal Theory}

Now we study variation of bulk viscosity coefficient $\eta$ and relaxation time $\tau$ with respect to the cosmic time. It has already been mentioned that for truncated theory $\varepsilon=0$ and hence Equation (13) reduces to

$$
\Pi+\tau \dot{\Pi}=-3 \eta H .
$$

In order to have exact solution of the system of equations one more physically plausible relation is required.

Thus, we consider the well known relation

$$
\tau=\frac{\eta}{\rho} .
$$

Using Equations (17), (20), (44) and (46) in Equation (45) one can obtain

$$
\begin{aligned}
\eta= & \frac{\left(C_{3} t^{1-n}-M_{1}\right)(M-U(t))^{-1}\left[B+C \exp \left(D t^{1-n}\right)\right]^{\frac{1}{\alpha+1}}+B\left[B+C \exp \left(D t^{1-n}\right)\right]^{\frac{-\alpha}{\alpha+1}}}{C_{4} t^{-n} \exp \left(D t^{1-n}\right)\left[B+C \exp \left(D t^{1-n}\right)\right]^{-2}+\frac{C_{3}(n-1) t^{n-2}}{M-U(t)}-\frac{C_{4}}{B \alpha} \frac{C_{3} t^{n-1}-M_{1}}{M-U(t)} \exp \left(D t^{1-n}\right)\left[B+C \exp \left(D t^{1-n}\right)\right]^{-1}} \\
& +\frac{C_{3} t^{n-1}-M_{1}}{a^{2}(M-U(t))^{2}}\left\{2 n t^{2 n-1} \exp \left(D t^{1-n}\right)-2 m_{1} t^{n} \exp \left(D t^{1-n}\right)\right\}+\frac{2 m_{1}+m_{2}}{t^{n}}
\end{aligned}
$$

where $C_{4}=B C \alpha\left(2 m_{1}+m_{2}\right)$.

(ii) Evaluation of Bulk Viscosity in Full Causal Theory

It has already been mentioned that for full causal theory $\varepsilon=1$ and hence Equation (13) reduces to

$$
\Pi+\tau \dot{\Pi}=-3 \eta H-\frac{\tau \Pi}{2}\left(3 H-\frac{\dot{\tau}}{\tau}-\frac{\dot{\eta}}{\eta}-\frac{\dot{T}}{T}\right)
$$

On the basis of Gibb's inerrability condition, Maartens [40] has suggested the equation of state for temperature as

$$
T \propto \exp \int \frac{\mathrm{d} p}{\rho+p},
$$

which with the help of Equation (16) gives

$$
T=T_{0}\left[1-B \rho^{-(\alpha+1)}\right]^{\frac{\alpha}{\alpha+1}} .
$$

using Equations (20), (40), (46) and (50) in Equation (48) one can obtain

$$
\Pi+\frac{\eta}{\rho} \dot{\Pi}=-\eta \frac{2 m_{1}+m_{2}}{t^{n}}-\frac{\eta \Pi}{2 \rho}\left[\frac{2 m_{1}+m_{2}}{t^{n}}-\frac{\dot{\rho}}{\rho}-\frac{\dot{T}}{T}\right],
$$

which on simplification yields the expression for bulk viscosity

$$
\begin{aligned}
\eta= & \frac{\left(M_{1}-C_{3} t^{1-n}\right)(M-U(t))^{-1}\left[B+C \exp \left(D t^{1-n}\right)\right]^{\frac{1}{\alpha+1}}-B\left[B+C \exp \left(D t^{1-n}\right)\right]^{\frac{-\alpha}{\alpha+1}}}{C_{4} t^{-n} \exp \left(D t^{1-n}\right)\left[B+C \exp \left(D t^{1-n}\right)\right]^{-2}+\frac{C_{3}(n-1) t^{n-2}}{M-U(t)}-\frac{C_{4}}{B \alpha} \frac{C_{3} t^{n-1}-M_{1}}{M-U(t)} \exp \left(D t^{1-n}\right)\left[B+C \exp \left(D t^{1-n}\right)\right]^{-1}} \\
& \left.+\frac{C_{3} t^{n-1}-M_{1}}{a^{2}(M-U(t))^{2}}\left\{2 n t^{2 n-1} \exp \left(D t^{1-n}\right)-2 m_{1} t^{n} \exp \left(D t^{1-n}\right)\right\}+\frac{2 m_{1}+m_{2}}{t^{n}}\right)\left(\frac{\left(2 m_{1}+m_{2}\right)(1-\alpha)}{t^{n}}-\frac{C_{2}\left(1+t^{-n}\right) \exp \left(D t^{1-n}\right)}{\left(B+C \exp \left(D t^{1-n}\right)\right)}\right) \\
& +\left(\frac{\left(C_{3} t^{n-1}-M_{1}\right)}{2(M-U(t))}+\frac{B}{2\left(B+C \exp \left(D t^{1-n}\right)\right)}\right)
\end{aligned}
$$




\section{Discussion}

In this paper we have studied bulk viscous Bianchi type III space-time geometry with generalized Chaplygin gas and time-varying gravitational and cosmological constants. We have obtained a new set of Einstein's equations by considering $\frac{\dot{R}_{1}}{R_{1}}=\frac{\dot{R}_{2}}{R_{2}}=\frac{m_{1}}{t^{n}}$ and $\frac{R_{3}}{R_{3}}=\frac{m_{2}}{t^{n}}$. In all cases energy density, bulk viscosity and cosmological constant are decreasing as gravitational constant $G(t)$ is increasing with time. Shear dies out with evolution of the universe for large value of $t$. For accelerating model of the universe, the deceleration parameter $q<0$ for $t<\left(\frac{2 m_{1}+m_{2}}{3 n}\right)^{\frac{1}{n-1}}$. We find that $\frac{\sigma}{\theta}=\frac{\sqrt{2}\left(m_{1}-m_{2}\right)}{\sqrt{3}\left(2 m_{1}+m_{2}\right)}=$ constant . Thus anisotropy is maintained throughout. However, if $m_{1}=m_{2}$, then $\frac{\sigma}{\theta}=0$, and then the model isotropizes. In case II for $n=1$, we have $H \propto \frac{1}{t}$ which is considered to be fundamental and match with the observations. In order to have clear idea of variation in behavior of cosmological parameters, relevant graphs have been plotted. All graphs of cosmological parameters go with cosmological observations.

\section{Acknowledgements}

S. K. would like to thank U. G. C. New Delhi for providing financial support under the scheme of major research project F. No. 41-765/2012 (SR). S. K. and R. K. would like to thank Inter University Centre for Astronomy and Astrophysics for providing facilities.

\section{References}

[1] Singh, T. and Singh, G.P. (1991) Bianchi Type V and Vi Cosmological Models in Lyra Geometry. Astrophysics and Space Science, 182, 189-200. http://dx.doi.org/10.1007/BF00644999

[2] Singh, T. and Singh, G.P. (1992) Bianchi Type III and Kantowski-Schas Cosmological Models in Lyra Geometry. International Journal of Theoretical Physics, 31, 1433-1446. http://dx.doi.org/10.1007/BF00673976

[3] Reddy, D.R.K., Santikumar, R. and Naidu, R.L. (2012) Bianchi Type III Cosmological Models in f(R, T) Theory of Gravity. Astrophysics and Space Science, 342, 249-252. http://dx.doi.org/10.1007/s10509-012-1158-7

[4] Pradhan A., Zia, R. and Amirhashchi, H. (2012) Anisotropic Bianchi Type III String Cosmological Models in Normal Gauge for Lyra’s Manifold with Electromagnetic Field. Bulgarian Journal of Physics, 39, 248-269.

[5] Singh, J.K. and Rani, S. (2015) Bianchi Type III Cosmological Models in Lyra’s Geometry in the Presence of Massive Scalar Field. International Journal of Theoretical Physics, 54, 545-560. http://dx.doi.org/10.1007/s10773-014-2247-x

[6] Caldwell, R.R. and Doran, M. (2004) Cosmic Microwave Background and Supernova Constraints on QuintessenceConcordance Regions and Target Models. Physical Review D, 69, Article ID: 103517. http://dx.doi.org/10.1103/physrevd.69.103517

[7] Huang, Z.Y., Abdalla, E. and Su, R.K. (2006) Holographic Explanation of Wide Angle Power Correlation Suppression in the Cosmic Microwave Background Radiation. JCAP, 13, Article ID: 0605. http://dx.doi.org/10.1088/1475-7516/2006/05/013

[8] Caldwell, R.R., Komp, W., Parker, L. and Daniel, A.T.V. (2006) A Sudden Gravitational Transition. Physical Review $D$, 73, Article ID: 023513. http://dx.doi.org/10.1103/PhysRevD.73.023513

[9] Daniel, S.F., Caldwell, R.R., Cooray, A. and Melchiorri, A. (2008) Large Scale Structure as a Probe of Gravitational Slip. Physical Review D, 77, Article ID: 103513. http://dx.doi.org/10.1103/physrevd.77.103513

[10] Fedeli, C., Moscardini, L. and Bartelmann, M. (2009) Observing the Clustering Properties of Galaxy Clusters in Dynamical Dark Energy Cosmologies. Astronomy Astrophysics, 500, 667-679. http://dx.doi.org/10.1051/0004-6361/200811477

[11] Peebles, P.J.E. and Ratra, B. (2003) The Cosmological Constant and Dark Energy. Reviews of Modern Physics, 75, 559. http://dx.doi.org/10.1103/revmodphys.75.559

[12] Hawking, S.W. (1969) On the Rotation of the Universe. Monthly Notices of the Royal Astronomical Society, 142, 129141. http://dx.doi.org/10.1093/mnras/142.2.129

[13] Hinshaw, G., Weiland, J.L., Hill, R.S., Odegard, N., Larson, D., et al. (2009) Five Year Wilkinson Microwave Aniso- 
tropy Probe (WMAP) Observations-Cosmological Interpretations. Astrophysical Journal Supplement Series, 180, 225. http://dx.doi.org/10.1088/0067-0049/180/2/225

[14] Collins, C.B. and Hawking, S.W. (1973) Why Is the Universe Isotropic? Astrophysical Journal, 180, 317-334. http://dx.doi.org/10.1086/151965

[15] Sahni, V. and Starobinski, A. (2000) The Case for a Positive Cosmological Lambda Term. International Journal of Modern Physics D, 9, 373-444.

[16] Dirac, P.A.M. (1937) The Cosmological Constants. Nature, 139, 323. http://dx.doi.org/10.1038/139323a0

[17] Canuto, V.M. and Narlikar, J.V. (1980) Cosmological Tests of the Hoyle-Narlikar Conformal Gravity. Astrophysical Journal, 6, 236.

[18] Turyshev, S.G. (2004) 35 Years of Testing Relativistic Gravity-Where Do We Go from Here? Lecture Notes in Physics, 648, 311-330. http://dx.doi.org/10.1007/978-3-540-40991-5_19

[19] Chakraborty, S. and Roy, A. (2008) Anisotropic Cosmological Models with Bulk Viscosity for Variable G and Lambda. Astrophysics and Space Science, 313, 389-392. http://dx.doi.org/10.1007/s10509-007-9708-0

[20] Singh, C.P. and Beesham, A. (2010) Anisotropic Bianchi Type V Perfect Fluid Space-Time with Variable G and Lambda. International Journal of Modern Physics A, 25, 3825-3834. http://dx.doi.org/10.1142/S0217751X10050123

[21] Singh, K.P. (2010) On Robertson-Walker Universe Model with Variable Cosmological Term and Gravitational Constant in Cosmological Relativity Theory. Turkish Journal of Physics, 34, 173-180.

[22] Khurshudyan, M., Mazhari, N.S., Momeni, D., Myrzakulov, R. and Raza, M. (2015) Observational Constrains on Model of the Universe with Time Variable Gravitational and Cosmological Constants Along MOG. International Journal of Theoretical Physics, 54, 484-505. http://dx.doi.org/10.1007/s10773-014-2242-2

[23] Ellis, G.F.R. (1979) In: Schas, R., Ed., General Relativity and Cosmology, Enrico Fermi Course, Academic Press, New York, 47.

Misner, C.W. (1968) The Isotropy of the Universe. Astrophysical Journal, 151, 431.

Hu, B.L. (1983) Vacuum Viscosity and Entropy Generation in Quantum Gravitational Processes in the Early Universe. In: Fang, E.J. and Ruttini, R., Eds., Advances in Astrophysics, World Scientific, Singapore.

[24] Fabris, J.C., Concalves, S.V.B. and de Sá Ribeiro, R. (2006) Bulk Viscosity during the Acceleration of the Universe. General Relativity and Gravitation, 38, 495-506. http://dx.doi.org/10.1007/s10714-006-0236-y

[25] Singh, C.P., Kumar, S. and Pradhan, A. (2007) Early Viscous Universe with Variable Gravitational and Cosmological Constant. Classical and Quantum Gravity, 24, 455-474. http://dx.doi.org/10.1088/0264-9381/24/2/011

[26] Singh, T. and Chaubey, R. (2007) Bianchi Type-V Universe with Viscous Fluid and Lambda-Term. Pramana, 68, 721734. http://dx.doi.org/10.1007/s12043-007-0072-y

[27] Singh, J.P. and Baghel, P.S. (2009) Bianchi Type V Cosmological Model with Constant Deceleration Parameter in General Relativity. International Journal of Theoretical Physics, 48, 449-462. http://dx.doi.org/10.1007/s10773-008-9820-0

[28] Singh, J.P. and Baghel, P.S. (2009) Bianchi Type V Cosmological Models with Time Dependent Lambda Term. Electronic Journal of Theoretical Physics, 6, 85-95.

[29] Kotambkar, S., Singh, G.P. and Kelkar, R.K. (2014) Anisotropic Cosmological Models with Quintessence. International Journal of Theoretical Physics, 53, 449-460. http://dx.doi.org/10.1007/s10773-013-1829-3

[30] Riess, A.G., Filippenko, A.V., Challis, P., Clocchiatti, A., Diercks, A., et al. (1998) Observational Evidence from Supernovae for an Accelerating Universe and a Cosmological Constant. Astronomical Journal, 116, 1009-1038. http://dx.doi.org/10.1086/300499

[31] Perlmutter, S., Aldering, G., Goldhaber, G., Knop, R.A., Nugent, P., et al. (1999) Measurements of Omega and Lambda from 42 High Redshift Supernovae. Astronomical Journal, 517, 565-586. http://dx.doi.org/10.1086/307221

[32] Dev, A., Alcaniz, J.S. and Jain, D. (2003) Cosmological Consequences of a Chaplygin Gas Dark Energy. Physical Review D, 67, Article ID: 023515. http://dx.doi.org/10.1103/physrevd.67.023515

[33] Sen, A.A. and Scherrer, R.J. (2005) Generating the Generalized Chaplygin Gas. Physical Review D, 72, Article ID: 063511. http://dx.doi.org/10.1103/PhysRevD.72.063511

[34] Debnath, U. (2011) Modified Chaplygin Gas with Variable G and Lambda. Chinese Physics Letters, 28, Article ID: 119801. http://dx.doi.org/10.1088/0256-307X/28/11/119801

[35] Bento, M.C., Bertolami, O. and Sen, A.A. (2002) Generalized Chaplygin Gas, Accelerated Expansion and Dark Energy Matter Unification. Physical Review D, 66, Article ID: 043507. http://dx.doi.org/10.1103/physrevd.66.043507

[36] Gorini, V., Kamenshchik, A. and Moschella, U. (2003) Can a Chaplygin Gas Be a Plausible Model for Dark Energy? Physical Review D, 67, Article ID: 063509. http://dx.doi.org/10.1103/physrevd.67.063509 
[37] Debnath, U., Banerjee, A. and Chakraborty, S. (2004) Role of Modified Chaplygin Gas in Accelerated Universe. Classical and Quantum Gravity, 21, 5609-5618. http://dx.doi.org/10.1088/0264-9381/21/23/019

[38] Dev, A., Alcaniz, J.S. and Jain, D. (2003) Cosmological Consequences of a Chaplygin Gas Dark Energy. Physical Review D, 67, Article ID: 023515. http://dx.doi.org/10.1103/physrevd.67.023515

[39] Paul, B.C., Thakur, D. and Verma, M.M. (2013) Observational Constraints on Modified Chaplygin Gas in HaravaLifshitz Gravity with Dark Radiation. Pramana, 81, 691-718. http://dx.doi.org/10.1007/s12043-013-0593-5

[40] Maartens, R. (1995) Dissipative Cosmologies. Classical and Quantum Gravity, 12, 1455-1465. http://dx.doi.org/10.1088/0264-9381/12/6/011

[41] Raychaodhuri, A.K. (1955) Relativistic Cosmology. Physical Review, 98, 1123.

http://dx.doi.org/10.1103/PhysRev.98.1123 